\title{
The Origins of Analytic Mechanics in the 18th century
}

\author{
Marco Panza
}

Last version before publication: H. N. Jahnke (ed.) A History of Analysis, American Mathematical Society and London Mathematical Society, 2003, pp. 137-153.

Up to the 1740s, problems of equilibrium and motion of material systems were generally solved by an appeal to Newtonian methods for the analysis of forces. Even though, from the very beginning of the century - thanks mainly to Varignon (on which cf. [Blay 1992]), Jean Bernoulli, Hermann and Euler - these methods used the analytical language of the differential calculus, and were considerably improved and simplified, they maintained their essential feature. They were founded on the consideration of a geometric diagram representing the mechanical system under examination, and consequently applied only to (simple) particular and explicitly defined systems. The possibility of expressing the conditions of equilibrium and motion of a general system of bodies, independently of its particular character, only arose when new and essentially non-Newtonian principles were advanced and employed. These principles - such as those of least action and of virtual velocitiesare generally known as "variational principles". In the second half of 18th century, mainly thanks to Lagrange, it was shown that these principles permitted the conditions of equilibrium and motion of a mechanical system to be expressed by a suitable system of equations. Though these equations differed when applied to different systems, their form was always the same, whatever the system they were concerned with. As long as this form could be expressed by some general equations, any problem of equilibrium and motion for a particular system could be interpreted as a particular case of one or the other of two general problems: the problem of equilibrium of a mechanical system, and the problem of motion of a mechanical system. Solving these 
problems was the same as solving two general equations, those expressing the conditions of equilibrium and motion of any system. Hence, the determination of these conditions for a particular system was simply a matter of specifying these equations and their solutions.

In the present chapter ${ }^{1}$, I sketch the history of this development, from the introduction of the first variational principles to the elaboration of Lagrange's "analytical mechanics". This is the history of the transformation of mechanics from a geometric science into an analytical one, or even, according to the Lagrange's dictum, into "a branch of analysis".

\section{The principle of the least action: Mauper- tuis, Euler and Lagrange (1740-1761)}

In the opening pages of his memoir "La loi du repos des corps" ([Maupertuis 1740]), Maupertuis explicitly contrasted the Newtonian principles of mechanics with another sort of principles, which would express, according to him, "the laws that Nature follows in certain combinations of circumstances" ("les Loix que la Nature suit dans certaines combinaisons de circonstances"; ibid., 170). Maupertuis mentioned, as two known examples of these latter principles, the principle of maximal descent of the center of gravity and the principle of conservation of vis viva. Moreover, he proposed a new principle of the same sort, which he called "the law of rest", expressing the conditions of equilibrium for a system of bodies acted upon by any number of central forces ${ }^{2}$ when these forces were directly proportional to any integral power-let us say $m$ - of the distance to their centers.

We consider a system of $n$ bodies and we write " $M_{i}$ " for the mass of the $i$-th body $(1 \leq i \leq n)$, and " $p_{i}$ ", " $q_{i}$ ", $\ldots$, " $w_{i}$ " for the distances of this body to the centers of the forces $P_{i}, Q_{i}, \ldots, W_{i}$, acting upon it. We then have:

$$
P_{i}=\mathcal{P}_{i} p_{i}^{m} \quad ; \quad Q_{i}=\mathcal{Q}_{i} q_{i}^{m} \quad ; \quad \ldots \quad ; \quad W_{i}=\mathcal{W}_{i} w_{i}^{m}
$$

where $\mathcal{P}_{i}, \mathcal{Q}_{i}, \ldots, \mathcal{W}_{i}$ are suitable constants of proportionality depending upon the particular nature of the forces. According to Maupertuis, these

\footnotetext{
${ }^{1}$ On the subject of the present chapter, cf.: [Panza 1991] and [Panza 1995]; [Grigorian 1965], [Szabo 1987], [Pulte 1989], [Barroso Filho 1994] and [Galletto 1990].

${ }^{2}$ Following the 18 th century terminology, I will use the term "force", without any other specification, to refer to accelerative forces, rather than motive forces.
} 
constants express the "intensities" of the respective forces. Maupertuis' law of rest asserts that the system is in equilibrium if the sum

$$
\sum_{i=1}^{n} M_{i}\left[\mathcal{P}_{i} p_{i}^{m+1}+\mathcal{Q}_{i} q_{i}^{m+1}+\ldots+\mathcal{W}_{i} w_{i}^{m+1}\right]
$$

is a maximum or a minimum.

Maupertuis deduced this condition from another one, namely:

$$
\sum_{i=1}^{n} M_{i}\left[P_{i} d p_{i}+Q_{i} d q_{i}+\ldots+W_{i} d w_{i}\right]=0
$$

The deduction was strictly speaking incorrect, since the condition $d Z=0$ is a necessary but not sufficient condition for $Z=\operatorname{Min} / \operatorname{Max}$. In any case, it makes it clear that Maupertuis' law reduces to the condition

$$
\sum_{i=1}^{n} M_{i}\left[\int P_{i} d p_{i}+\int Q_{i} d q_{i}+\ldots+\int W_{i} d w_{i}\right]=\operatorname{Min} / \operatorname{Max}
$$

Even though Maupertuis did not remark upon it, equation (1) expresses a principle already advanced by Jean Bernoulli in a letter to Varignon of January, 26th 1711, and known as the principle of virtual velocities (cf. [Varignon 1725], t. II, 174-176). The differentials $d p_{i}, d q_{i}, \ldots, d w_{i}$ in this equation express what Bernoulli called the "virtual velocities" of the forces $P_{i}, Q_{i} \ldots, W_{i}$, while the products $P_{i} d p_{i}, Q_{i} d q_{i}, \ldots, W_{i} d w_{i}$ were generally referred to in 18th century as the "moments" of forces $P_{i}, Q_{i} \ldots, W_{i}$. From a physical point of view, the moment of a force was intended to represent the elementary (that is infinitesimal) component of force with respect to its direction. If we pass from accelerative to motive forces, by introducing as a factor the mass of the body upon which the force acts, and consider the products $M_{i} P_{i} d p_{i}, M_{i} Q_{i} d q_{i}, \ldots, M_{i} W_{i} d w_{i}$, we have what in modern terms is the "virtual work" performed by the (motive) forces $M_{i} P_{i}, M_{i} Q_{i},, \ldots, M_{i} W_{i}$. Hence, the equation (1) corresponds to the modern principle of "virtual work" and the integrals

$$
\begin{aligned}
& (m+1) \int M_{i} P_{i} d p_{i}=M_{i} \mathcal{P}_{i} p_{i}^{m+1} \\
& (m+1) \int M_{i} Q_{i} d q_{i}=M_{i} \mathcal{Q}_{i} q_{i}^{m+1} \\
& \cdots \\
& (m+1) \int M_{i} W_{i} d w_{i}=M_{i} \mathcal{W}_{i} w_{i}^{m+1}
\end{aligned}
$$


entering Maupertuis' principles are just proportional to the "work" that the (motive) forces $M_{i} P_{i}, M_{i} Q_{i}, \ldots, M_{i} W_{i}$ perform upon the body of mass $M_{i}$.

Since Bernoulli's principle does not assume that the forces are proportional to an integral power of the distance to their centers, the new principle of Maupertuis is less general. However, there is something new and important in Maupertuis' principle. This is the idea of expressing the condition of equilibrium as a condition of maximum or minimum of a single general expression.

Although this idea would prove to be very fruitful, mathematically, it seems that Maupertuis was primarily interested in connecting the conditions of equilibrium with a principle of economy (that is a final cause) operating in natural phenomena. He made this clear in a subsequent memoir ([Maupertuis 1744]). This memoir deals with the refraction of light, but concerns the science of nature as a whole, since the question is that of showing that the law of refraction - which was well known at that time - perfectly agrees with "another that nature must follow still more inviolably" ("une autre que la Nature doit suivre encore plus inviolablement"; ibid., 418), according to which "Nature, in making its effects, always acts by the simplest means possible" ("la Nature, dans la production de ses effets, agit toujours par les moyens les plus simples"; ibid, 421). In Maupertuis' new interpretation, the trajectory of light in refraction is precisely the one "for which the quantity of action is the least" ("par lequel la quantité d'action est la moindre"), such a quantity being "proportional to the sum of the distances each multiplied by the speed with which the body moves through them" ("proportionnelle à la somme des espaces multipliés chacun par la vitesse avec laquelle le corps les parcourt"; ibid., 423). If $s_{1}$ and $s_{2}$ are the distances covered by the light-rays in two contiguous media, and $v_{1}$ and $v_{2}$ the respective speeds, this implies that the sum $s_{1} v_{1}+s_{2} v_{2}$ will be a minimum. It follows that its differential must be equal to zero. It is easy to verify that the (Fermat's ) law of refraction is a consequence of this hypothesis. This rather modest success convinced Maupertuis that he had found a very general law of nature, namely a final cause for natural phenomena.

However, Maupertuis' metaphysical enthusiasm blinded him to an important difficulty. Even though we might think that the example of refractionas well as the deduction of the law of rest-provides evidence for asserting that nature always acts according to an aim of minimization (or maximization), it remains to be shown how to express this aim in the form of a general equation. Although Maupertuis claimed to deal with all natural phenomena, 
he did not provide a general method for dealing with the equilibrium and motion of a general system, in either the 1740 or the 1744 memoir.

A different judgment applies to the two appendices in Euler's Methodus inveniendi ([Euler 1744]), where Euler discussed the possibility of solving some mechanical problems by referring to a general principle asserting that "absolutely nothing happens in the world, in which some condition of maximum or minimum does not reveal itself" ("nihil omnino in mundo contingint, in quo non maximi minimive ratio quæpiam eluceat"; ibid., $245^{3}$ ). I shall consider here the second of these appendices, devoted to the motion of an isolated body subject to central forces. Of course, Euler was not interested in finding the law of this motion, which was well known by that time. Instead, he wanted to compare the Newtonian derivation of this law with a different derivation based on the search for a maximum or a minimum. His aim was to determine the integral form $\mathcal{F}$, such that the condition $\mathcal{F}=$ Max/Min entails this law. This gives a formal expression for the metaphysical notion of natural economy ([Galletto 1990], 91): in modern terms, Euler assumed the existence of a Lagrangian for the motion of an isolated body subject to conservative central forces, and tried to calculate it. Having justified his hypothesis by means of rather weak metaphysical arguments, Euler discussed some examples. Let us consider one of these: the motion of a body in vacuo subject to an initial speed and a variable central force.

Euler started from the hypothesis that the trajectory of the body is the one which minimizes the integral $\int M d s \sqrt{h}=\int M h d t$, where $M$ is the mass of the body and $h=\frac{2 f x}{M}$ is proportional to the distance $x$ that a body of mass $M$ attracted by a constant motive force $f$ and starting from the rest must cover in order to attain speed $v$ (cf. [Euler 1736], t. I, 80). Since $\sqrt{h}=v$, Euler's hypothesis is equivalent to the condition

$$
\int \frac{1}{2} M v d s=\int \frac{1}{2} M v^{2} d t=M i n
$$

The product $M v$ is Descartes' "quantity of motion", or, in Euler's terminology "the quantity of motion in any place". By multiplying it by the "spatiolum" $d s$, Euler obtains what he calls "the collective motion of the body through space $d s "$ ("motum corporis collectivum per spatiolum $d s "$;[Euler 1744], app. II. par. 2. 311-312). According to Euler's hypothesis,

\footnotetext{
${ }^{3}$ The previous formulation is taken from the first appendix, where the principle is applied to the study of elastic deformation. On this appendix cf. [Truesdell 1960], 199225 and [Fraser 1983], 199.
} 
the trajectory has to minimize the "aggregate" of all motions, taken in this way. Since $\frac{1}{2} M v^{2}$ is nothing but kinetic energy, or "vis viva", as people called it in the 17th and 18th centuries, this hypothesis states that in the motion under consideration, kinetic energy is conserved over time.

Euler had proved in his Mechanica ([Euler 1736], II, prop. 23, 92-93), that if the force is decomposed into two orthogonal components $X=X(x)$ and $Y=Y(x)$, then $d h=-X d x-Y d y$ and thus $h=K-\int X d x-\int Y d y$, where $K$ is a constant of integration. By substituting this value in Euler's hypothesis, $\int d s \sqrt{h}=\operatorname{Min}$, we obtain:

$$
\begin{gathered}
\int d s \sqrt{K-\int X d x-\int Y d y}= \\
\int d x \sqrt{\left[1+\left(\frac{d y}{d x}\right)^{2}\right]\left[K-\int X d x-\int Y d y\right]}=\text { Min }
\end{gathered}
$$

By applying the mathematical method presented in the main body of his treatise ${ }^{4}$ to such a condition, Euler deduced that the equation of the trajectory is a parabola if $X$ and $Y$ are constant, and an ellipse if $X=2 x$ and $Y=2 y$, as it is easy to verify by applying Newtonian methods. This proves that we arrive at the same conclusion whether we use these methods or start from Euler's hypothesis. The integral form $\mathcal{F}$ that enters the condition $\mathcal{F}=\operatorname{Max} /$ Min referred to the motion of a free body in vacuo subject to an initial speed and a variable central force (that is the Lagrangian of such a simple system) is just $\int M d s \sqrt{h}$.

Clearly, Euler's argument is not independent of the Newtonian methods. However, these are only used to transform the hypothesis advanced by Euler into the condition (2). If this condition is accepted as a particular case of the principle, asserting that in the motion of a free body the integral $\int M d s \sqrt{h}=$ $\int M h d t$ is a minimum, then the derivation reduces to a particular application of Euler's new mathematical method of maximis and minimis. Nevertheless, this is neither a proof of any mechanical principle, nor a justification for generalizing Euler's hypothesis to the motion of an arbitrary system. In the final part of this appendix, Euler merely deduced his condition by using a Newtonian analysis of forces in the case of the motion of an isolated body, the speed of which depends only on its position, and then asserted that it remains true when more bodies are concerned. Not only did he leave his

\footnotetext{
${ }^{4}$ Cf. chapter 12 , par. 3 in this book.
} 
assertion without any real justification (by confining himself to an appeal to metaphysics), but he also failed to explain what form his principle should take when it refers to the motion of more than one body.

However, we observe that a straight-forward generalization of his principle to the case of $n$ bodies provides a condition on their motion which is formally similar to Maupertuis' law of rest. In fact, if $\Psi_{i}$ is the total force acting upon the $i$-th body and $\psi_{i}$ its distance from the center of force, Maupertuis' law says that under suitable conditions, the equilibrium of the system is given by the condition $\sum_{i=1}^{n} M_{i} \int \Psi_{i} d \psi_{i}=\operatorname{Max} / M i n$., while Euler's principle suitably generalized states that the motion of the same system is given by the condition $\sum_{i=1}^{n} M_{i} \int v_{i} d s_{i}=\operatorname{Max} / \operatorname{Min}$.

Euler pointed out this analogy in a letter to Maupertuis of December 10th, 1745 (cf [Brunet 1938], 61-62 and [Euler Op], ser. IV-A, VI, 56-58). But where Euler saw a mathematical analogy, Maupertuis saw a confirmation of the tendency to minimize action in nature. In subsequent memoir ([Maupertuis 1746]), Maupertuis asserted that the second appendix of Euler's Methodus inveniendi was, in fact, an application of the more general principle that he had advanced in 1744 and that, he now called, for the first time, the "principle of the least quantity of action". According to this principle, "when a change happens in Nature, the quantity of action necessary for the change is as small as possible" ("lors qu'il arrive quelque changement dans la Nature, la Quantité d'Action, nécessaire pour ce changement, est la plus petite qu'il soit possible"), the quantity of action being "the product of the masses of the bodies by its speed and the distances covered" ("le produit de la Masse des Corps, par leur vîtesse et par l'espace qu'ils parcourent" ibid., 290). Of course, Maupertuis gave no justification of his principle. He just appealed to divine wisdom, and to the consideration of three simple examples: the impact of hard and elastic bodies and the equilibrium of a lever. This is particularly unsatisfactory since these examples are chosen for their simplicity and do not provide a suitable basis for obtaining a formula expressing the quantity of action in general.

This difficulty was explicitly noted by Euler in a letter to Maupertuis of May 24th, 1746, (cf. [Brunet 1938], 62-65, particularly 62 and [Euler Op]., ser. IV-A, VI, 63-65 and 69-71, particularly 63-64), where he admitted that he did not understand "how the consideration of the distance covered in a given time necessarily enters the determination of the quantity of action" 
("comment la considération de l'espace parcouru dans un temps donné doit entrer dans la determination de la quantité d'Action"). He observed that in Maupertuis' examples this distance is always expressed by the speed itself, but no argument is given to show that this is generally true ${ }^{5}$. Euler's remark foreshadows a research program that would engage him between 1748 and 1751 , in an attempt to answer the following question: is the condition $\int M v d s=$ Max $/$ Min (giving the motion of a free body animated by central forces) a particular case of a more general condition, describing not only the motion of any system of bodies in a field of central forces, but also the solution of a larger class of mechanical problems?

Euler started ([Euler 1748a]) with a generalization of the classical problem of the catenary: to find the curve formed by a perfectly flexible string (a chain) "acted upon by arbitrary forces" ("sollicité par des forces quelconques"), when it is in equilibrium. Although he adopted Maupertuis' term "action", he explicitly stated that it did not denote a definite natural entity, but a generally indeterminate mathematical quantity that "can always be represented by a certain algebraic formula" ("pourra toujours être représentée par une certaine formule algébrique"; ibid., 150). In order to determine this formula, Euler began by using an analysis of force to find the differential equation giving the solution of the problem, and then looked for an integral form $\int \mathcal{A}$, such that the condition $\int \mathcal{A}=\operatorname{Min} /$ Max is equivalent to such an equation, so that the form $\int \mathcal{A}$ expresses the action.

To accomplish the first task, he regarded the string as being composed of infinitesimal elements, each of them acted upon by infinitesimal forces, and reduced these forces to two orthogonal components directed along the direction of two axes. Then he considered a generic element of the string as being fixed, so that the two orthogonal components of the forces acting upon any other anterior element of the string tend to rotate the string in two opposite directions around this element. By applying a principle analogous to the principle of angular momentum ("although the string remains in equilibrium, because it is perfectly flexible, the moments of all the forces acting $[\ldots]$ on the anterior part of the string [...] necessarily cancel each other out"; "afin que le fil demeure en équilibre, puisqu'il est parfaitement flexible, il

\footnotetext{
${ }^{5}$ Euler's remark makes clear the mathematical reasons of the well-known querelle about Maupertuis' priority in the statement of the principle of the least action, involving, from 1749, many mathematicians and philosophers. On this querelle cf.: [Montucla 1799-1802], III, 648-54; [Brunet 1929], 128-158 and [Brunet 1938], 8-26; [Gueroult 1934], 215-235; [Dugas 1950], 256-262; and [Fleckenstein 1957], XXV-XLVI.
} 
faut que les momens de toutes les forces, dont la partie antérieure du fil [...] est sollicitée [...] se détruisent mutuellement", ibid., 153) and by integrating over the particles of the string, he obtained the equation $d y \int X d s-d x \int Y d s$, where $X d x$ and $Y d y$ are the orthogonal components of the infinitesimal force acting upon the particle with coordinate $x$ and $y$. Euler's goal was to derive this equation from a condition of maximis or minimis. To this end, it is necessary to know the particular nature of the functions $X$ and $Y$.

Let us consider the case where the string is acted upon by any number of external central forces expressed by a function of the distance from their centers, so that the orthogonal components of the total force acting upon an element of the string are $X=\sum_{j=1}^{\mu} \frac{\Lambda_{j} x_{j}}{\lambda_{j}}$ and $Y=\sum_{j=1}^{\mu} \frac{\Lambda_{j} y_{j}}{\lambda_{j}}$, where $\Lambda_{j}, \lambda_{j}$, $x_{j}$ and $y_{j}(j=1, \ldots, m)$ are the (accelerative) forces acting upon this element, the distances of it from the centers of these forces and the orthogonal coordinates of these centers, respectively. By substituting these expressions in the previous identity, differentiating and integrating, Euler deduced the condition:

$$
\frac{\frac{d^{2} y}{d x^{2}}}{1+\left(\frac{d y}{d x}\right)^{2}}\left[\sum_{j=1}^{\mu} \int \Lambda_{j} d \lambda_{j}\right]=\sum_{j=1}^{\mu} \frac{\Lambda_{j}\left(y_{j}-\frac{d y}{d x} x_{j}\right)}{\lambda_{j}}
$$

Euler then showed that this equation can also be deduced from the condition

$$
\int d s \sum_{j=1}^{\mu} \int \Lambda_{j} d \lambda_{j}=\operatorname{Max} / \operatorname{Min}
$$

Thus, the quantity of action is in this case expressed by the integral form $\int d s \sum_{j=1}^{\mu} \int \Lambda_{j} d \lambda_{j}$

The analogy with the condition expressing Maupertuis' law of rest is evident: if we denote by $\Omega_{i}$ the sum $\int P_{i} d p_{i}+\ldots+\int W_{i} d w_{i}$ of the integrals of the moments of forces acting upon the $i$-th body of the Maupertuis system, by $M_{i}$ the mass of this body, and finally by $\Omega$ the sum $\sum_{j=1}^{\mu} \int \Lambda_{j} d \lambda_{j}$ of the forces acting upon the elastic string, that is the sum the moment of the forces acting upon an element of this string), we have the conditions $\int d s \Omega=$ Max/Min and $\sum_{i=1}^{n} M_{i} \Omega_{i}=\operatorname{Max} / \operatorname{Min}$ for the equilibrium of the elastic string and of the Maupertuis system of bodies, respectively.

In another memoir([Euler 1748b]), Euler clarified this analogy by searching for a formulation of Maupertuis' law of rest in the case of the equilibrium 
of a fluid mass (that is a continuous system of particles attracted towards fixed centers by central forces proportional to the distance from their centers). Following the same plan as the previous memoir, Euler first solved this problem using a Newtonian analysis of forces, by assuming that the equilibrium of the fluid mass is only possible if the total force acting upon any particle is orthogonal to the surface of this mass. Since this implies the condition $\sum_{j=1}^{\mu} \int \Lambda_{j} d \lambda_{j}=K$ - that is $d\left(\sum_{j=1}^{\mu} \int \Lambda_{j} d \lambda_{j}\right)=0$-(where $K$ is a constant and $\Lambda_{j}$ and $\lambda_{j}(j=1, \ldots, m)$ are again the forces acting upon a certain particle of the fluid mass and the distances of this particle from the centers of these forces), he concluded that the quantity of action is here expressed by $\Omega=\sum_{j=1}^{\mu} \int \Lambda_{j} d \lambda_{j}$.

In this way, Euler showed that the sum of integrals $\Omega$ that appears in the equation of equilibrium of Maupertuis' system of bodies also appears in the equation of equilibrium of a fluid mass and an elastic string (under the specified conditions). Also observed that the same expression appears in the equation of motion of a free body attracted by central forces, since if $\Lambda_{j}$ and $\lambda_{j}$ $(j=1, \ldots, m)$ are the forces acting upon this body and its distances from the centers of these forces, respectively, then the condition $\int M v d s=M a x / M i n$, announced in 1744, is equivalent to the condition $\int M \Omega d t=$ Max/Min. In fact $\sum_{j=1}^{\mu} \Lambda_{j} d \lambda_{j}$ is equal to the moment of the total force acting upon the body, which we can express by the product $-F d s$ (where the distance $s$ is taken in the direction of the force $F$, which tends to diminish it). It follows that

$$
\sum_{j=1}^{\mu} \Lambda_{j} d \lambda_{j}=-F d s=-\frac{d v}{d t} d s=-d v \frac{d s}{d t}=-v d v
$$

and thus $\Omega=K-\frac{1}{2} v^{2}$ and:

$$
\int \Omega d t=\int\left(2 K-v^{2}\right) \frac{d t}{2}=K t-\frac{1}{2} \int v d s
$$

The results of the two memoirs of 1748 were synthesized by Euler in two memoirs of 1751 ([Euler 1751a] and [Euler 1751b]; on these memoirs cf. [Fraser 1983], 200-203). In the first one, he tried to show the "harmony" between Maupertuis' law of rest - which he now formulated for any system of bodies attracted by central forces - and a generalization of his 1744 principle 
to any system of bodies, i. e. a version of Maupertuis' principle of least action. We have already seen that the law of rest can be expressed by the condition $\sum_{i=1}^{n} M_{i} \Omega_{i}=\operatorname{Max} / \operatorname{Min}$. Here, $\Omega_{i}$ is the sum of the integrals of the moments of forces $P_{i}, \ldots, W_{i}$, which are supposed to be proportional to any integral power of the distance to their centers. Euler now considered a generalized version of this law, where $\Omega_{i}$ expresses the sum $\sum_{j=1}^{\mu} \int \Lambda_{j, i} d \lambda_{j, i}$ of the integrals of the moments of forces $\Lambda_{j, i}(j=1,2, \ldots, \mu)$ acting upon the $i$-th body, whatever these forces are. We have also seen that the condition $\int M v d s=$ Max $/$ Min for the motion of a free body of mass $M$ attracted by a central force, is equivalent to the condition $\int M \Omega d t=M a x / M i n$, because the product $M \Omega$ - which, in Euler's terminology is the "effort" of the body with mass $M$-is equal to $M K-M \frac{1}{2} v^{2}$, that is a constant minus the vis viva of the same body (or, in modern terminology, its kinetic energy). This is the law of conservation of vis viva. Thus, in order to prove that the "harmony" between the conditions $\sum_{i=1}^{n} M_{i} \Omega_{i}=\operatorname{Max} /$ Min and $\int M \Omega d t=$ Max $/$ Minwhich is apparent because of the occurrence of the integral form $\Omega$ - extends to an analogous "harmony" between the generalized law of rest and the principle of least action (that is a generalisation, to any system of moving bodies attracted by central forces, of the 1744 principle concerned with the motion of a free body attracted by a central force and entailing the condition $\int M v d s=\operatorname{Max}(\operatorname{Min})$, it is enough to prove that this law of conservation holds when the "effort" and the vis viva refer to any system of moving bodies attracted by central forces. Euler only proved that this is true for a system composed by two bodies linked by a rod and attracted towards a common center, but he claimed "that the same demonstration extends to any sorts of bodies attracted together to as many centers of force as one wishes" ("que la même démonstration s'étend, tant à autant de corps liés ensemble qu'à au-tant de centers de forces qu'on voudra" [Euler 1748a], 179).

Although the "harmony" that he has shown in this way does not imply that the principle of least action can be deduced from the law of rest, when these principles are referred to any system of bodies, Euler seems to conclude that, in order to found mechanics on a single principle of maximinis or minimis, it is necessary only to produce a proof of the generalized law of rest. The aim of the second memoir of 1751 is to provide such a proof. However, Euler's derivation is merely a metaphysical argument founded on the representation of a central force by a rowing-machine and on the assumption that 
"toute force agit autant qu'elle peut" ([Euler 1751b], 248).

Euler's failure to found mechanics on a single principle does not affect his essential result. This is that the integral form $\Omega=\sum_{j=1}^{\mu} \int \Lambda_{j} d \lambda_{j}$ plays an essential role when we try to express the motion or the equilibrium of different sorts of mechanical systems. From Euler's point of view, this is just a form, a sort of analytical invariant. The fact that from a modern point of view we see behind this form a notion that is very close to the notion of potential energy, only shows that, historically, the mathematical expression of potential energy came before than the corresponding physical notion.

It was against this background that the young Lagrange began to be interested in the principle of least action and the foundation of mechanics. Born in Turin January 25th, 1736, he was at this time 18 years old, but confident enough to write to Euler June 28th, 1754 ([Lagrange Oevr], XIV, 135-138) to communicate some results on differentiation and to announce his studies of the problem of maximis and minimis and its application to mechanics. Thanks to these studies, in 1755 Lagrange became a professor at the School of Artillery in Turin, where he taught until 1766. In that year, he left Turin (where in 1757 he had helped to found the Privata Società Scientifica, which later became the Turin Academy of Sciences in 1783) for Berlin. He had been called there by Frederick the Great, at the suggestion of d'Alembert, to succeed Euler as director of the class of Mathematics of the Academy of Sciences. He stayed in Berlin until 1787, when, after the death of Frederick, he decided to move to Paris to enter the French Academy of Science. He died there April 10th, 1813 (for Lagrange's life consult [Burzio 1942] and [Sarton 1944]).

Lagrange presented the results of his studies into the problem of maximis and minimis and its application to mechanics in two memoirs in 1761 ([Lagrange 1761a] and [Lagrange 1761b] ). In the first of these memoirs ${ }^{6}$, Lagrange provided a new formulation of Euler's method of maximis and minimis in terms of his new $\delta$-formalism. This was the first version of the modern calculus of variations (cf. ch. 12 of this book).

In his second memoir ${ }^{7}$, Lagrange stated that the solution of "all the prob-

\footnotetext{
${ }^{6}$ On this memoir cf. [Goldstine 1980], 110-129, [Fraser 1985a], 155-172 and [Dahan 1990], 81-88.

${ }^{7} \mathrm{On}$ the foundation of mechanics proposed by Lagrange in the second memoir of 1761 cf., apart from works mentioned in the footnote (1) below, [Fraser 1983], [Barroso Filho and Comte 1988], [Dahan 1990], and [Galletto 1990], 112-121.
} 
lems of dynamics" ("toutes les questions de dynamique" [Lagrange 1761b], 196) can be obtained by a suitable application of a quasi-algebraic procedure founded on an application of the method of undetermined coefficients, which is in turn made possible by the mutual independence of the variations $\delta d^{i} \alpha_{j}$, where the $\alpha_{j}$ are any number of variables of position. According to Lagrange, this was made possible by the assumption that Euler's principle of 1744 applies to the motion of any system of $n$ of bodies attracted by internal or external central forces expressed by any function of the distance between the centers of these forces and the body upon which they act. This principle states that the motion of a system of this sort is subject to the condition $\sum_{i=0}^{n} \int M_{i} v_{i} d s_{i}=\operatorname{Max} . /$ Min. For Lagrange, this means that it satisfies the variational equation

$$
\begin{aligned}
\delta\left(\sum_{i=0}^{n} \int M_{i} v_{i} d s_{i}\right) & =\delta \int\left(\sum_{i=0}^{n} M_{i} v_{i} d s_{i}\right)=\int \delta\left(\sum_{i=0}^{n} M_{i} v_{i} d s_{i}\right) \\
& =\int \delta\left[\sum_{i=0}^{n}\left(M_{i} v_{i} \delta d s_{i}\right)+\sum_{i=0}^{n}\left(M_{i} v_{i} \delta v_{i}\right) d t\right]=0
\end{aligned}
$$

Because of the mechanical nature of the problem, it is not possible to consider the variations $\delta d s_{i}$ and $\delta v_{i}$ as mutually independent. Thus, in order to apply his method, Lagrange has to transform the previous equation into another one, where only variables of position occur. In order to do that, he appealed to the condition of conservation of vis viva. Using this condition and some easy analytical manipulations, (5) becomes:

$$
\int\left\{\begin{array}{l}
\sum_{i=0}^{n} M_{i}\left[d\left(v_{i} \frac{d x_{i}}{d s_{i}}\right) \delta x_{i}+d\left(v_{i} \frac{d y_{i}}{d s_{i}}\right) \delta y_{i}+d\left(v_{i} \frac{d z_{i}}{d s_{i}}\right) \delta z_{i}\right] \\
+\sum_{i=0}^{m}\left[M_{i}\left(P_{i} \delta p_{i}+Q_{i} \delta q_{i}+\ldots+W_{i} \delta w_{i}+\delta \Gamma\right)\right] d t
\end{array}\right\}=0
$$

where $x_{i}, y_{i}$ and $z_{i}$ are the Cartesian orthogonal coordinates of the $i$-th body, $P_{i}, Q_{i}, \ldots, W_{i}$ are the external (accelerative) forces acting upon it, $p_{i}, q_{i}$, $\ldots, w_{i}$ are the distances of the body from the centers of these forces and $\Gamma$ is the sum of all the terms like $M_{i} M_{j} \int P_{i, j} d p_{i, j}$ arising from the internal forces. By expressing both the distances $p_{i}, q_{i}, \ldots, w_{i}$ and the distances between two distinct bodies occurring in $\Gamma$ in terms of the variables $x_{i}, y_{i}$ and $z_{i}$ and substituting into (6), Lagrange obtained an equation of the form

$$
\sum_{i=0}^{n} \mathcal{X}_{i} \delta x_{i}+\mathcal{Y}_{i} \delta y_{i}+\mathcal{Z}_{i} \delta z_{i}=0
$$


The method of undetermined coefficients can be applied to such an equation only if it is possible to consider all the variables $x_{i}, y_{i}$ and $z_{i}$ as mutually independent, that is: if the system is free from any internal constraint. If this is not the case a new reduction is necessary, consisting in replacing the dependent variables by their values given by the equations of condition. In both the first and the second case, the application of the method of undetermined coefficients is the final stage of Lagrange's method, since it provides a sufficient number of equations for expressing the motion of all the bodies of the system.

For the modern reader, Lagrange's second memoir essentially contains the first clear formulation of the principle of the least action and provides a mathematical technique for working with it. This does not seem to have been Lagrange's own evaluation of his work. According to him the essential achievement of his memoir was the reduction of the solution of the problem of motion for any system of bodies to a quite simple application of the quasialgebraic principle of undetermined coefficients. Lagrange's future researches in that domain were aim to justify this reduction in a simpler way.

\section{The Analytical Mechanics ${ }^{8}$}

\subsection{The principle of virtual velocities: Jean Bernoulli, d'Alembert and Lagrange}

According to a letter Lagrange wrote to Euler October 28th, 1762 ([Lagrange Oevr], XIV, 198-199), his two memoirs of 1761 were only a short abstract of a larger treatise that he had written, or had planned to write ([Galletto 1990], 112113). It is possible that one of the reasons why Lagrange just presented a short abstract is the fact that a new and different analytical foundation of mechanics had occurred to him (ibid., 139-149). Lagrange presented his new conception in a memoir ([Lagrange 1764]), written to answer a question advanced in 1762 by the Academy of Paris, concerning the libration of the moon. More than 15 years later ([Lagrange 1780]), he outlined a more elaborate and general version of the same program was outlined by Lagrange. He finally realised this program in the Méchanique analitique ([Lagrange 1788]).

\footnotetext{
${ }^{8}$ On the subject of this section, cf. [Panza 1991] and [Panza 1995], § 3; [Galletto 1990], 149-179 and [Barroso Filho 1994], 137-303.
} 
Lagrange's idea has two clear sources. The first is the Bernoullian principle of virtual velocities for the equilibrium of any system of bodies animated by central forces. Let us imagine that the system is subject to a small rectilinear motion and, for any force acting upon each body, let us decompose the motion of this body into two orthogonal motions, one of which is taken in the same direction as this force. Jean Bernoulli expressed the latter motion by a speed he called the "virtual velocity of the force". The principle announced by Jean Bernoulli says that the system is in equilibrium when the total sum of the products of the motive forces and their virtual velocities is equal to zero. The second source is d'Alembert's principle for dynamics. In his Traité de Dynamique ([d'Alembert 1743]; on d'Alembert's treatise cf. [Fraser 1985b] and [Truesdell 1960], 186-188), d'Alembert based statics on a particular version of the Bernoullian principle of virtual velocities ([d'Alembert 1743], 37) and then presented a method for reducing any dynamic problem to an associated problem of equilibrium. This method consisted in decomposing the real motion of any body in the system into two virtual motions: the motion that is communicated from the outside, and another motion that is the difference between the latter motion and the real motion of this body. The first of these motions is then composed of the real motion and a virtual motion that is destroyed by the mutual actions of bodies and the constraints of the system. Since the virtual motion balances these actions and constraints, it can be calculated by examining the conditions of equilibrium of the system. Thus, if we know the motion that is communicated from the outside, we can determine the real motion of the system by examining the conditions of equilibrium.

Lagrange's idea is even simpler than d'Alembert's : the equilibrium of a system results from the balance of two opposite total forces, the applied force and the inertial reaction (which can be regarded as two different mathematical expressions of the same force). Thus, if a system of $n$ bodies is given, where $P_{i}, Q_{i}, \ldots, W_{i}$ are the central forces acting upon the $i$-th body, we consider the virtual system, where these forces act together with $3 n$ orthogonal inertial forces $X_{i}, Y_{i}$ and $Z_{i}$. Then, by appealing to a suitable version of the principle of virtual velocities, we can derive a condition of equilibrium for such a virtual system and obtain the general equation of motion for the given system. Lagrange expressed the virtual velocities of the forces - or, in his terminology, the virtual velocities "evaluated along the direction of the forces" - in terms of variations of the distance between the body upon which these forces act and their centers. The general equation of motion of a system 
of $n$ bodies is then the following:

$$
\sum_{i=1}^{n} M_{i}\left(\frac{d^{2} x}{d t^{2}} \delta x_{i}+\frac{d^{2} y}{d t^{2}} \delta y_{i}+\frac{d^{2} z}{d t^{2}} \delta z_{i}+P_{i} \delta p_{i}+Q_{i} \delta q_{i}+\ldots+W_{i} \delta w_{i}\right)=0
$$

a fundamental relation known today as d'Alembert's principle.

According to Lagrange, two procedures can be used to derive the equations of motion of the system from this relation. We can simply express the variables $p_{i}, q_{i}, \ldots, w_{i}$ by means of the variables $x_{i}, y_{i}$ and $z_{i}$, eliminate those variables which depend on other ones, according to the internal constraints of the system, and apply the method of undetermined coefficients. Or, we can express the variables occurring in (7) in terms of a suitable number $\nu$ of variables $\varphi_{i}$ (the generalized variables, as we call them today). Lagrange showed ([Lagrange 1780], 218-220 and [Lagrange 1788], 216-227) that, if the forces are conservative, then (7) gives rise to the equation

$$
\sum_{i=1}^{\nu}\left[d \frac{\delta T}{\delta d \varphi_{i}}-\frac{\delta T}{\delta \varphi_{i}}+\frac{\delta U}{\delta \varphi_{i}}\right] \delta \varphi_{i}=0
$$

where $T$ and $U$ are what we would now call the kinetic and the potential energy of the system. This is the original version of the so-called Lagrangian equation of motion. Its deduction from (7) depends on the choice of the variables $\varphi_{i}$. We must ensure that the variations of these variables are mutually independent. Thus, we have to chose these variables in a suitable way, subject to the equations of condition of the system. Since this could be difficult, Lagrange presented a way of making this elimination easier in the Méchanique analitique. This device is now known as the method of Lagrange multipliers ([Lagrange 1788], 44-58 and 227-32). The basic idea is to express the internal constraints of the system by adding to the first term of (8) a suitable term for any equation of condition. If this equation is $\Psi=0$, this term have to be of the form $\psi \delta \Psi$, where $\psi$ is an indeterminate multiplier to be determined by analytical procedures.

The Lagrangian equation of motion and the method of Lagrange multipliers are important contributions to theoretical mechanics. However, they appear in both the 1780 memoir on the libration of the moon and in the Méchanique analitique as tools to make possible an application of the quasialgebraic method of undetermined coefficients to the general equation expressing the principle of virtual velocities, in order to deduce from it the equations of motion for any system of bodies. The essence of Lagrange's 
program in the foundation of dynamics after 1764 was rather to use the principle of virtual velocities, regarded as a variational principle, in order to reduce dynamics to a formal deduction of the differential equations of motion, by means of the method of undetermined coefficients.

Of course, the same program can be applied to statics, starting with a suitable version of the principle of virtual velocities. This is the object of the first part of the Méchanique analitique, the second part being is devoted to dynamics.

\subsection{Mechanics in Lagrange's Théorie des fonctions analytiques}

In his 1761 memoirs and in his works on mechanics, Lagrange defined variations as infinitesimal differences or increments. He also made use of this definition in justifying his inferences and drawing out his proofs. This attitude has led historians to consider these works as essentially opposed, in their mathematical and epistemological approach, to the Théorie des fonctions analytiques ([Lagrange 1797]; on the first part of Lagrange's treatise cf. [Panza 1992], ch. III. 6), where Lagrange proposed a new and noninfinitesimal interpretation of the calculus (cf. chapter 4) and traced the broad outline of an application of his theory to the foundation of mechanics ([Lagrange 1797], 223-277; 2nd ed., 311-381). I disagree with this view.

It seems to me that Lagrange's employment of infinitesimal calculus in mechanics before 1797 is largely consistent with the non-infinitesimal foundation of mechanics proposed in the Théorie. In fact what is essential in Lagrange's employment of variations in the memoirs of 1761, 1764 and 1780 and in the Méchanique analitique is not the infinitesimal character of the variations, but the mutual independence of some of them. In the Théorie, Lagrange simply replaced mutually independent variations by suitable independent functions that express finite virtual velocities. What is new in this later treatise is the aim of founding mechanics on purely analytical (that is non-variational, or generally non-mechanical) principles. In order to do so, i. e. to reduce mechanics to a "branch of analysis", Lagrange had either to eliminate any reference to a non-analytical principle such as the principles of virtual velocities, or to derive this principle by analytical means from a non-mechanical starting point. Instead, Lagrange derived the equations of motion without any explicit appeal to the principle of virtual velocities, and 
he did so in such a way that the fundamental relation (7) is a simple corollary of his result. In the second edition of his treatise ([Lagrange 1797], 2nd ed., 352-357) he also proves the static version of this principle analytically. Nevertheless, it is obvious in both cases that what is proved analytically is simply an identity which is formally equivalent to the principle, since no purely analytical consideration could be sufficient to prove that such an identity expresses the physical conditions of a system of bodies.

Lagrange assumed that motion is expressed mathematically by a functional relationship between space and time, that is by a function $s=f(t)$. By appealing to his general results about the development of any function in a power series, he proved ([Lagrange 1797], 226-227) that for any function $G(t, \vartheta)$ of the form $g_{1}(t) \vartheta+g_{2}(t) \vartheta^{2}$ (expressing the distance covered after a time $\vartheta$ by a uniformly accelerated motion with acceleration $2 g_{2}(t)$ and initial speed $\left.g_{1}(t)\right)$ and for any $t$, we can take $\vartheta$ small enough for the function $F(t, \vartheta)=f^{\prime}(t) \vartheta+\frac{1}{2} f^{\prime \prime}(t) \vartheta^{2}$ to approximate the given function $s=f(t)$ closer than $G(t, \vartheta)$-where $f^{\prime}(t)$ and $\frac{1}{2} f^{\prime \prime}(t)$ are respectively the first and the second coefficient of the development of the difference $f(t+\vartheta)-f(t)$ in a power series. Using this result, he concluded that the first two derivatives $f^{\prime}(t)$ and $f^{\prime \prime}(t)$ provide a measure of the instantaneous speed and the instantaneous acceleration (or accelerative force) of the motion $s=f(t)$, respectively, and therefore express them.

Let $\tau$ be a variable expressing time and $t$ a value of $\tau$. Any curvilinear motion $\mathcal{M}$ expressed by the function $s=s(t)$ can be regarded as composed of three rectilinear motions, along the directions of the orthogonal axes $x$, $y, z$, expressed by the functions $x=x(t), y=y(t)$ and $z=z(t)$. Thus, the instantaneous speed $v(t)$ and the instantaneous acceleration $\gamma(t)$ at time $t$ of the motion $\mathcal{M}$ are given in terms of the instantaneous speeds and accelerations of the orthogonal motions composing it. The latter speeds are expressed by the functions $X=\left[x^{\prime}(t)\right] \tau, Y=\left[y^{\prime}(t)\right] \tau$ and $Z=\left[z^{\prime}(t)\right] \tau$, and the corresponding accelerations by the functions $X=\left[x^{\prime \prime}(t)\right] \tau^{2}, Y=\left[y^{\prime \prime}(t)\right] \tau^{2}$ and $Z=\left[z^{\prime \prime}(t)\right] \tau^{2}$. Hence we have:

$$
\begin{aligned}
& v(t)=\sqrt{\left[x^{\prime}(t)\right]^{2}+\left[y^{\prime}(t)\right]^{2}+\left[z^{\prime}(t)\right]^{2}} \\
& \gamma(t)=\sqrt{\left[x^{\prime \prime}(t)\right]^{2}+\left[y^{\prime \prime}(t)\right]^{2}+\left[z^{\prime \prime}(t)\right]^{2}}
\end{aligned}
$$

Since any motion can be regarded as composed of any number $\mu$ of other motions, we can also regard $\mathcal{M}$ as composed of $\mu$ curvilinear motions $\mathcal{M}_{1}, \ldots$, $\mathcal{M}_{\mu}$ expressed by $\mu$ functions, $s_{1}=s_{1}(t), \ldots, s_{\mu}=s_{\mu}(t)$ and its acceleration 
(or accelerative force) in $t$ as composed of $\mu$ accelerations (or accelerative forces) $g_{1}(t), \ldots, g_{\mu}(t)$. Since each of these motions can be regarded in turn as composed of three rectilinear motions along the directions of the $x, y$ and $z$ axes, $\mathcal{M}$ can be regarded as composed of $3 \mu$ rectilinear motions expressed by the functions $x_{j}=x_{j}(\tau), y_{j}=y_{j}(\tau)$ and $z_{j}=z_{j}(\tau)$, and its acceleration (or accelerative force) in $t$ as composed of the accelerations expressed by the functions $X_{j}=\frac{1}{2}\left[x_{j}^{\prime \prime}(t)\right] \tau^{2}, Y_{j}=\frac{1}{2}\left[y_{j}^{\prime \prime}(t)\right] \tau^{2}$ and $Z_{j}=\frac{1}{2}\left[z_{j}^{\prime \prime}(t)\right] \tau^{2}(j=1, \ldots, \mu)$.

By eliminating $\tau$ between the equations $X=\left[x^{\prime}(t)\right] \tau, Y=\left[y^{\prime}(t)\right] \tau$ and $Z=\left[z^{\prime}(t)\right] \tau$ we obtain two equations expressing a straight line $r$. By eliminating $\tau$ between the equations $X_{j}=\frac{1}{2}\left[x_{j}^{\prime \prime}(t)\right] \tau^{2}, Y_{j}=\frac{1}{2}\left[y_{j}^{\prime \prime}(t)\right] \tau^{2}$ and $Z_{j}=\frac{1}{2}\left[z_{j}^{\prime \prime}(t)\right] \tau^{2}$, we obtain two equations expressing a straight line $r_{j}$. If $\mathcal{X}$, $\mathcal{Y}, \mathcal{Z}$ and $\mathcal{X}_{j}, \mathcal{Y}_{j}, \mathcal{Z}_{j}(j=1, \ldots, \mu)$ are the angles formed by the straight lines $r$ and $r_{j}$, with the axis $x, y$ and $z$, simple geometric considerations gave:

$$
\begin{array}{ll}
x^{\prime}(t)=v(t) \cos \mathcal{X} \quad ; \quad x^{\prime \prime}(t)=\sum_{j=1}^{\mu} x_{j}^{\prime \prime}(t)=\sum_{j=1}^{\mu} \gamma_{j}^{\prime \prime}(t) \cos \mathcal{X}_{j} \\
y^{\prime}(t)=v(t) \cos \mathcal{Y} \quad ; \quad y^{\prime \prime}(t)=\sum_{j=1}^{\mu} y_{j}^{\prime \prime}(t)=\sum_{j=1}^{\mu} \gamma_{j}^{\prime \prime}(t) \cos \mathcal{Y}_{j} \\
z^{\prime}(t)=v(t) \cos \mathcal{Z} \quad ; \quad z^{\prime \prime}(t)=\sum_{j=1}^{\mu} z_{j}^{\prime \prime}(t)=\sum_{j=1}^{\mu} \gamma_{j}^{\prime \prime}(t) \cos \mathcal{Z}_{j}
\end{array}
$$

and

$$
\cos \Delta_{j}=\cos x \cos \mathcal{X}_{j}+\cos y \cos \mathcal{Y}_{j}+\cos z \cos \mathcal{Z}_{j}
$$

where $\Delta_{j}$ is the angle formed by the directions of the speed $v(t)$ and the acceleration $\gamma_{j}(t)$. Combining (10) and (11) we obtain

$$
x^{\prime \prime}(t) \cos \mathcal{X}+y^{\prime \prime}(t) \cos \mathcal{Y}+z^{\prime \prime}(t) \cos \mathcal{Z}=\sum_{j=1}^{\mu} \gamma_{j} \cos \Delta_{j}
$$

and so:

$$
x^{\prime \prime}(t) x^{\prime}(t)+y^{\prime \prime}(t) y^{\prime}(t)+z^{\prime \prime}(t) z^{\prime}(t)-\sum_{j=1}^{\mu} v(t) \gamma_{j}(t) \cos \Delta_{j}=0
$$

which is the dynamic equation of motion of any free body (of unit mass) acted upon by the forces $\gamma_{j}(t)(j=1, \ldots, \mu)$. 
By applying the method of Lagrange multipliers, we can express the internal constraints of a system of bodies in terms of indeterminate forces. Equation (13) can then be transformed, by a suitable analytical procedure ([Lagrange 1797], 251-356; 2nd ed., 350-357), to give the equation of motion of every body belonging to any system. These equations have thus been deduced formally without any explicit reference to the principle of virtual velocities $^{9}$. Moreover a general relation formally equivalent to (7)—which expresses this principle in the Méchanique analitique - can be deduced from (13) by linear composition and introduction of masses (that is by passing to motive forces):

$$
\sum_{i=1}^{n} M_{i}\left[x_{i}^{\prime \prime}(t) x_{i}^{\prime}(t)+y_{i}^{\prime \prime}(t) y_{i}^{\prime}(t)+z_{i}^{\prime \prime}(t) z_{i}^{\prime}(t)+\sum_{j=1}^{\mu} \gamma_{i, j}(t)\left(-v_{i}(t) \cos \Delta_{i, j}\right)\right]=0
$$

where the index $i$ refers to the different bodies in the system and the index $j$ refers to the different forces acting upon each of these bodies.

Although Lagrange's belief that he had proved the principle of virtual velocities analytically is certainly illusory, the foundation of mechanics sketched in the Théorie des fonctions analytiques makes apparent his effort to reduce the role of non-Newtonian principles in mechanics and identify the subject with a quasi-algebraic deductive system based on a general Newtonian analysis of forces.

This shows the difference between Lagrange's interpretation of his own results and any modern evaluation of them. Where a modern reader, knowing the future developments of classical mechanics and in particular Hamilton's achievements, sees a formidable stock of variational principles, methods and formulas, Lagrange simply saw the possibility of returning, if from a novel analytical point of view, to Newton's approach. In a sense, the 18th century seems to end without a real awareness of its essential contribution to the evolution of modern mechanics: from Lagrange's own point of view the adjective "analytical" in "analytical mechanics", was more important than the noun "mechanics".

\footnotetext{
${ }^{9}$ As it is presented in the first edition of the Théorie this procedure needs an implicit appeal to the static version of such a principle. This is the reason why Lagrange gives an "analytical proof" of this principle in the second edition.
} 


\section{References}

[d'Alembert 1743] J. B. le R. d'Alembert, Traité de dynamique, David, Paris 1743; IInd. ed., 1758.

[Barroso Filho 1994] W. Barroso Filho, La mécanique de Lagrange. Principes et Methodes, Karthala, Paris, 1994.

[Barroso Filho and Comte 1988] W. Barroso Filho and C. Comte, "La formalisation de la dynamique par Lagrange (1736-1813)", in R. Rasched (ed. by), Sciences à l'époque de la révolution française, Libr. Sci. A. Blanchard, Paris 1988, pp. 329-48.

[Blay 1992]

M. Blay, La naissance de la mechanique analytique. La science du mouvement au tournat des XVIIème et XVIIIème siècle, PUF, Paris. 1992.

[Brunet 1929]

P. Brunet, Maupertuis. I. Etude biographique. II. L'ouvre et sa place dans la pensée scientifique et philosophique du XVIIIe siècle, Hermann, Paris 1929.

[Brunet 1938]

P. Brunet, Etude historique sur le principe de la moindre action, Hermann, Paris 1938.

[Burzio 1942]

F. Burzio, Lagrange, UTET, Torino, 1942.

[Dahan 1990]

A. Dahan-Dalmedico, "Le formalism variationnel dans les travaux de Lagrange", supplement to the number 124, 1990, of Atti della Accademia delle Scienze di Torino, Classe di Scienze Fisiche, Matematiche e Naturali (proceedings of the meeting hold in Paris, College de France, September 27th-29th, 1988), pp. 81-106. 
[Dugas 1950]

[Euler Op]

[Euler 1736]

[Euler 1744]

[Euler 1748a]

[Euler 1748b]

[Euler 1751a]

[Euler 1751b]
R. Dugas, Histoire de la mecanique, Dunod, Paris and Ed. du Griffon, Neuchâtel, 1950.

L. Euler, Opera Omnia, publ. par la Soc. Sci. Nat. Helveticæ, Leipzig-Berlin-Basel, $1911-\ldots$

Mechanica, sive motus scientia analytice exposita, ex typ. Acad. sci. imp., Petropoli, 1736 (2 vols.); in [Euler Op], ser. II, I and II.

L. Euler, Methodus inveniendi lineas curvas maxime minimive proprietate gaudentes, M. M. Bousquet et Soc., Lausanæ et Genevæ, 1744; in [Euler Op], ser. I, XXIV.

L. Euler, "Recherches sur les plus grands et les plus petits qui se trouvent dans les actions des forces", Hist. Acad. Roy. Sci. et Bell. Lett. [de Berlin], Mém., 4, 1748 [pub. 1750], pp.149-88; in [Euler Op], ser. I, V, 1-37.

L. Euler, "Réflexions sur quelques loix générales de la nature qui s'observent dans les effets des forces quelconques" Hist. Acad. Roy. Sci. et Bell. Lett. [de Berlin], Mém., 4, 1748 [pub. 1750], pp. 183-218; in [Euler Op], ser. I, V. 38-63.

L. Euler, "Harmonie entre les principes generaux de repos et de mouvement de M. de Maupertuis", Hist. Acad. Roy. Sci. et Bell. Lett. [de Berlin], Mém., 7, 1751 [pub. 1753], pp. 169-98; in [Euler Op], ser. I, V. 152-172.

L. Euler, "Essay d'une démomstration métaphysique du principe générale de 
l'équilibre", Hist. Acad. Roy. Sci. et Bell. Lett. [de Berlin], Mém., 7, 1751 [pub. 1753], pp. 246-54; in [Euler Op], ser. II, V, $250-256$.

[Fleckenstein 1957]

[Fraser 1983]

[Fraser 1985a]

[Fraser 1985b]

[Galletto 1990]

[Goldstine 1980]

[Grigorian 1965]
J. O. Fleckenstein, "Vorwort des Herausgebers" of vol. V, ser. II of [Euler Op], ivi, pp. VII-LI.

C. Fraser, "J. L. Lagrange's Early Contributions to the Principles and Methods of Mechanics", Arch. Hist. Exact Sci., 28, 1983, pp. 197-241.

C. Fraser, "J. L. Lagrange's Changing Approach to the Foundations of the Calculus of Variations", Arch. Hist. Exact Sci., 32, 1985, pp. 151-91.

C. Fraser, "D'Alembert's Principle: The Original Formulation and Application in Jean d'Alembert's Traité de Dynamique (1743)", Centaurus, 28, 1985, pp. 31-61 and 145-59.

D. Galletto, "Lagrange e la Meccanica Analitica", Memorie dell' Istituto Lombardo, Classe di Sci. Mat. e Nat., 29, 1990, pp. 77-179.

H. H. Goldstine, A History of the Calculus of Variations from the 17th through the 19th Century, Springer, New York, Heidelberg, Berlin, 1980.

A. T. Grigorian, "On the development of variational principles of mechanics", Archives internationales d'histoire des sciences, 18, 1965, pp. 23-35. 
[Gueroult 1934]

[Lagrange Oevr]

[Lagrange 1761a]

[Lagrange 1761b]

[Lagrange 1764]

[Lagrange 1780]
M. Gueroult, Dynamique et Métaphysique leibniziennes, Publ. de la Fac, des Lettres de l'Univ. de Strasbourg, Fasc. 68, Strasbourg 1934.

J. L. Lagrange, Euvres de Lagrage, publiées par les soins de M. J.-A. Serret et G: Darboux, Gauthier-Villars, Paris, 18671892 (14 vols.).

J. L. Lagrange, "Essai d'une nouvelle méthode pour déterminer les maxima et les minima des formules intégrales indéfinies", Mélanges de Philosophie et de Mathématiques de la Soc. Roy. de Turin, 2, 1760-61, IIe partie, pp. 173-95; in [Lagrange Oevr], I, 33-362.

J. L. Lagrange, "Applications de la méthode exposée dans le mémoir précédent à la solution de différents problèmes de dynamique", Mélanges de Philosophie et de Mathématiques de la Soc. Roy. de Turin 2, 1760-61, Ile partie, pp. 196-298, in [Lagrange Oevr], I, 363-468.

J. L. Lagrange, "Recherches sur la libration de la lune, dans lesquelles on tâche de résoudre la Question proposée par l'Académie Royale des Sciences pour le Prix de l'année 1764", Recueil des pieces qui ont remporté les prix de l'Acad. Roy. des Sci. [de Paris], 9, piec. de 1764, '65, '66, '70, '72 (pub. 1777), pp. 1-50; in [Lagrange Oevr], VI, 561.

J. L. Lagrange, "Théorie de la libration de la lune, et des autres Phénomenes qui dépendent de la figure non sphérique de 
[Lagrange 1788]

[Lagrange 1797]

[Maupertuis 1740]

[Maupertuis 1744]

[Maupertuis 1746]

[Montucla 1799-1802] cette Planète", Nouv. Mém. Acad. Roy. Sci. et Bell. Lett. [de Berlin], 1780 (pub. 1782), classe de Math., pp. 203-309; in [Lagrange Oevr], V, 5-122.

J. L. Lagrange, Méchanique analitique, La veuve Desaint, Paris 1788; 2nd ed., Mecanique analytique, Courcier, Paris, 1811-1815 (2 vols.) [the 3th ed. edited by M. Bertrand is reproduced in [Lagrange Oevr], $\mathrm{XI}$ and XII].

J. L. Lagrange, Théorie des fonctions analytiques [...], Impr. de la Rép., Paris an V [1797]; 2nd. ed., Courcier, Paris 1813; in [Lagrange Oevr], IX.

P. L. M. Maupertuis, "Loi du repos des corps", Hist. Acad. Roy. Sci. [de Paris], Mém. Math. Phy., 1740 [publ. 1742; pres. 20/2/1740], pp. 170-76; in [Euler Op], ser, II, V, 268-273.

P. L. M. Maupertuis, "Accord de différentes Loix de la Nature aui avoient jusqu'ici paru incompatibles", Hist. Acad. Roy. Sci. [de Paris], Mém. Math. Phy., 1744 [publ. 1748; prés le 15/4/1744], pp. 417-26; in [Euler Op], ser, II, V, 274-281.

P. L. M. Maupertuis, "Le Loix du mouvement et du repos déduites d'un Principe Métaphysique", Hist. Acad. Roy. Sci. et Bell. Lettr. [de Berlin], Mém., 2, 1746 [publ. 1748], pp. 267-94; in [Euler Op], ser, II, V, 282-302.

J. E. Montucla, Histoire des Mathématiques, IIth. ed., H. Agasse, Paris, 1799-1802 (4 vols.). 
[Panza 1991]

[Panza 1992]

[Panza 1995]

[Pulte 1989]

[Sarton 1944]

[Szabo 1987]

[Truesdell 1960]
M. Panza, "The Analytic Foundation of Mechanics of Discrete Systems in Lagrange's Théorie des Fonctions Analytiques, Compared with Lagrange's Earlier Treatements of This Topic", Historia Scientiarum, 44, 1991, pp. 87-132 and 45, 1992, pp. 181-212.

M. Panza, La forma della quantita. Analisi algebrica e analisi superiore: il problema dell'unità della matemetica nel secolo dell'Illuminismo, Cahiers d'Histoire et de Philosophie des Sciences, 38 and 39, 1992.

M. Panza, "De la Nature épargnante aux Forces généreuses: le principe de Moindre Action entre mathématiques et métaphysique.Maupertuis et Euler, 17401751", Revue d'histoire des Sciences, 1995, 48, 435-520.

H. Pulte, Das Prinzip der kleinsten Wirkung und die Kraftkonzeptionen der rationalen Mechanik, Studia Leibnitiana, Sond. 19, F. Steiner Verlag, Stuttgart 1989.

G. Sarton, "Lagrange's Personality (17361813)", Proceedings of the American Society, 88, 1944, 457-496.

I. Szabó, Geschichte der mechanischen Prinzipien, Dritte, korrigierte und erweiterte Auflage, heraus. von P. Zimmermann und E. A. Felmann, Birkhäuser, Basel, Boston, Stutgart, 1987.

C. Truesdell, The Rational Mechanics of Flexible or Elastic Bodies (1638-1788), vol. $\mathrm{XI}, 2$ of the ser. II of [Euler Op]. 
[Varignon 1725]

P. Varignon, Nouvelle mécanique ou statique, C. Jombert, Paris 1725 (2 vols). 